American Journal of Applied Sciences 3 (4): 1790-1794, 2006

ISSN 1546-9239

(c) Science Publications, 2005

\title{
On Concircular Structure Spacetimes II
}

\author{
A. Shaikh and Kanak Kanti Baishya \\ Department of Mathematics, University of Burdwan, Golapbag, Burdwan-713104, West Bengal, India
}

\begin{abstract}
We studied concircular structure spacetimes which are connected 4-dimensional Lorentzian concircular structure manifolds.
\end{abstract}

2000 Mathematics subject classification: $53 \mathrm{C} 40,53 \mathrm{C} 50,53 \mathrm{C} 80$

Key words: $(\mathrm{CS})_{4}$-spacetime, perfect fluid spacetime, scalar curvature, conformally flat

\section{INTRODUCTION}

Recently the first author introduced the notion of Lorentzian concircular structure manifolds (briefly (LCS $)_{\mathrm{n}}$-manifolds) by citing an example of dimension $4^{[6]}$. Then in $^{[7]}$ the present authors studied its several applications to general relativity and physics. In this study we extend the study of ${ }^{[7]}$ and investigate some other interesting applications to relativity and cosmology. After preliminaries, we study perfect fluid non-flat $(\mathrm{CS})_{4}$-spacetimes and proved that if in such a spacetime the square of the length of the Ricci-operator is $(1 / 3) r^{2}$, then the spacetime can not contain pure matter and also in such a spacetime the pressure of the fluid is positive for $\alpha^{2}>\rho$ and negative for $\alpha^{2}>\rho, \alpha, \rho$ being non-zero scalars associated with the $(\mathrm{CS})_{4}$-spacetime. Section 4 is concerned with $(\mathrm{CS})_{4}$-spacetimes whose energy-momentum tensor is a Codazzi tensor and it is shown that in such a spacetime both the energy density and pressure of the fluid are constants over a hypersurface. Among others it is proved that if the energy-momentum tensor of a perfect fluid (CS) $)_{4}$-spacetime is a Codazzi tensor, then the possible local cosmological structure of the spacetime is of Petrov type I, D or O and also it is shown that if a perfect fluid $(\mathrm{CS})_{4}$-spacetime with divergence-free conformal curvature tensor admits a conformal Killing vector field then the spacetime is either conformally flat or of Petrov type N. The last section deals with a conformally flat $(\mathrm{CS})_{4}$-spacetime and proved that such a spacetime is infinitesimally spatially isotropic relative to the unit timelike vector field $\xi$.

Preliminaries: An n-dimensional Lorentzian manifold $\mathrm{M}$ is a smooth connected paracompact Hausdorff manifold with a Lorentzian metric $\mathrm{g}$, that is, $\mathrm{M}$ admits a smooth symmetric tensor field $\mathrm{g}$ of type $(0,2)$ such that for each point $\mathrm{p} \in \mathrm{CM}$, the tensor $\mathrm{g}_{\mathrm{p}}: \mathrm{T}_{\mathrm{p}} \mathrm{M} \times \mathrm{T}_{\mathrm{p}} \mathrm{M} \rightarrow \mathrm{R}$ is a non-degenerate inner product of signature $(-,+, \ldots,+)$, where $\mathrm{T}_{\mathrm{p}} \mathrm{M}$ denotes the tangent vector space of $\mathrm{M}$ at $\mathrm{p}$ and $\mathrm{R}$ is the real number space. $\mathrm{A}$ non-zero vector $\mathrm{v} \in \mathrm{T}_{\mathrm{p}} \mathrm{M}$ is said to be timelike (resp., non-spacelike, null, spacelike) if it satisfies $g_{\mathrm{p}}(\mathrm{v}, \mathrm{v})<0(\mathrm{resp}, \leq 0,=0,>0)^{[3]}$. The category to which a given vector falls is called its causal character.

Let $\mathrm{M}^{\mathrm{n}}$ be a Lorentzian manifold admitting a unit timelike concircular vector field $\xi$, called the characteristic vector field of the manifold. Then we have:

$$
\mathrm{g}(\xi, \xi)=-1
$$

Since $\xi$ is a unit concircular vector field, there exists a non-zero 1 -form $\eta$ such that for:

$$
\mathrm{g}(\mathrm{X}, \xi)=\eta(\mathrm{X})
$$

the equation of the following form holds:

$$
\left(\nabla_{\mathrm{X}} \eta\right)(\mathrm{Y})=\alpha\{\mathrm{g}(\mathrm{X}, \mathrm{Y})+\eta(\mathrm{X}) \eta(\mathrm{Y})\}(\alpha \neq 0)
$$

for all vector fields $\mathrm{X}, \mathrm{Y}$ where $\nabla$ denotes the operator of covariant differentiation with respect to the Lorentzian metric $g$ and $\alpha$ is a non-zero scalar function satisfies:

$$
\nabla_{\mathrm{x}} \alpha=(\mathrm{X} \alpha)=\alpha(\mathrm{X})=\rho \eta(\mathrm{X})
$$

$\rho$ being a certain scalar function. If we put:

$$
\phi X=\frac{1}{\alpha} \nabla_{X} \xi
$$

then from (3) and (5) we have:

$$
\phi \mathrm{X}=\mathrm{X}+\eta(\mathrm{X}) \xi,
$$

from which it follows that $\phi$ is a symmetric $(1,1)$ tensor. Thus the Lorentzian manifold $\mathrm{M}^{\mathrm{n}}$ together with the unit timelike concircular vector field $\xi$, its associated 1 -form $\eta$ and $(1,1)$ tensor field $\phi$ is said to

Corresponding Author: A. Shaikh, Department of Mathematics, University of Burdwan, Golapbag, Burdwan-713104, West Bengal, India 
be a Lorentzian concircular structure manifold (briefly $(\mathrm{LCS})_{\mathrm{n}}$-manifold $)^{[4]}$. In a (LCS $)_{\mathrm{n}}$-manifold, the following relations hold ${ }^{[4]}$ :

$$
\begin{gathered}
\text { a) } \eta(\xi)=-1, \text { b) } \phi \xi=0, \text { c) } \eta(\phi X)=0 \\
\text { d) } g(\phi X, \phi Y)=g(X, Y)+\eta(X) \eta(Y) \\
\eta(R(X, Y) Z)=\left(\rho-\alpha^{2}\right)[g(Y, Z) \eta(X)-g(X, Z) \eta(Y)](8) \\
S(X, \xi)=(n-1)\left(\rho-\alpha^{2}\right) \eta(X) \\
R(X, Y) \xi=\left(\rho-\alpha^{2}\right)[\eta(Y) X-\eta(X) Y]
\end{gathered}
$$

for any vector fields $\mathrm{X}, \mathrm{Y}, \mathrm{Z}$ where $\mathrm{R}, \mathrm{S}$ denote respectively the curvature tensor and the Ricci tensor of the manifold. General relativity flows from Einstein's equation given by:

$$
\mathrm{S}(\mathrm{X}, \mathrm{Y})-(\mathrm{r} / 2) \mathrm{g}(\mathrm{X}, \mathrm{Y})+\lambda \mathrm{g}(\mathrm{X}, \mathrm{Y})=\mathrm{kT}(\mathrm{X}, \mathrm{Y})
$$

for all vector fields $\mathrm{X}, \mathrm{Y}$ where $\mathrm{S}$ is the Ricci tensor of the type $(0,2), r$ is the scalar curvature, $\lambda$ is the cosmological contant, $\mathrm{k}$ is the gravitational constant and $\mathrm{T}$ is the energy momentum tensor of type $(0,2)$.

The energy momentum tensor $\mathrm{T}$ is said to describe a perfect fluid $^{[3]}$ if

$$
\mathrm{T}(\mathrm{X}, \mathrm{Y})=(\sigma+\mathrm{p}) \mathrm{A}(\mathrm{X}) \mathrm{A}(\mathrm{Y})+\mathrm{pg}(\mathrm{X}, \mathrm{Y})
$$

where $\sigma$ is the energy density function, $p$ is the isotropic pressure function of the fluid, $\mathrm{A}$ is a non-zero 1 -form such that $g(X, U)=A(X)$ for all $X, U$ being the flow vector field of the fluid.

In a $(C S)_{4}$-spacetime by considering the characteristic vector field $\xi \xi$ of the spacetime as the flow vector field of the fluid, the energy momentum tensor takes the form:

$$
\mathrm{T}(\mathrm{X}, \mathrm{Y})=(\sigma+\mathrm{p}) \eta(\mathrm{X}) \eta(\mathrm{Y})+\operatorname{pg}(\mathrm{X}, \mathrm{Y})
$$

The above results will be used in the next sections.

Perfect fluid non-flat (CS $)_{\mathbf{4}}$-spacetimes: In this section we consider that the matter distribution of a non-flat $(\mathrm{CS})_{4}$-spacetime be perfect fluid with $\sigma$ and $\mathrm{p}$ as its density and pressure respectively and the characteristic vector field $\xi$ of the spacetime as the flow vector field of the fluid. We take Einstein's field equation without cosmological constant. Then (11) can be written as:

$$
\mathrm{S}(\mathrm{X}, \mathrm{Y})-(\mathrm{r} / 2) \mathrm{g}(\mathrm{X}, \mathrm{Y})=\mathrm{kT}(\mathrm{X}, \mathrm{Y})
$$

From (13) and (14) we have:

$\mathrm{S}(\mathrm{X}, \mathrm{Y})-(\mathrm{r} / 2) \mathrm{g}(\mathrm{X}, \mathrm{Y})=\mathrm{k}[(\sigma+\mathrm{p}) \eta(\mathrm{X}) \eta(\mathrm{Y})+\mathrm{pg}(\mathrm{X}, \mathrm{Y})](15)$
Taking a frame field and contracting (15) over X and $\mathrm{Y}$ we obtain:

$$
\mathrm{r}=\mathrm{k}(\sigma-3 \mathrm{p})
$$

In view of (16), (15) yields:

$$
\mathrm{S}(\mathrm{X}, \mathrm{Y})=\mathrm{k}\left[\begin{array}{c}
(\sigma+\mathrm{p}) \eta(\mathrm{X}) \eta(\mathrm{Y}) \\
+\frac{1}{2}(\sigma-\mathrm{p}) \mathrm{g}(\mathrm{X}, \mathrm{Y})
\end{array}\right]
$$

Let $\mathrm{Q}$ be the Ricci operator i.e., $\mathrm{g}(\mathrm{QX}, \mathrm{Y})=\mathrm{S}(\mathrm{X}, \mathrm{Y})$. Then setting $\mathrm{X}=\mathrm{QX}$ in (17) we get:

$$
\mathrm{S}(\mathrm{QX}, \mathrm{Y})=\mathrm{k}\left[\begin{array}{c}
(\sigma+\mathrm{p}) \eta(\mathrm{QX}) \eta(\mathrm{Y}) \\
+\frac{1}{2}(\sigma-\mathrm{p}) \mathrm{S}(\mathrm{X}, \mathrm{Y})
\end{array}\right]
$$

Contracting (18) over $\mathrm{X}$ and $\mathrm{Y}$ we have:

$$
\|\mathrm{Q}\|^{2}=\mathrm{k}\left[(\sigma+\mathrm{p}) \mathrm{S}(\xi, \xi)+\frac{1}{2}(\sigma-\mathrm{p}) \mathrm{r}\right]
$$

Using (16) and (9) ( for $\mathrm{n}=4$ ) in (19) we obtain:

$$
\|\mathrm{Q}\|^{2}=\mathrm{k}\left[\begin{array}{c}
(\sigma+\mathrm{p})(-3)\left(\mathrm{p}-\alpha^{2}\right) \\
+\frac{1}{2}(\sigma-\mathrm{p}) \mathrm{k}(\sigma-3 \mathrm{p})
\end{array}\right]
$$

Again setting $\mathrm{X}=\mathrm{Y}=\xi$ in (17) we get:

$$
-3\left(p-\alpha^{2}\right)=\frac{k}{2}(\sigma+3 p)
$$

Since the $(\mathrm{CS})_{4}$-spacetime under consideration is non-flat, we have $\left(\rho-\alpha^{2}\right) \neq 0$ and hence, $(21)$ implies that: $(\sigma+3 p) \neq 0$ as $k \neq 0$ By virtue of (21) we obtain from (20) that:

$$
\|\mathrm{Q}\|^{2}=\mathrm{k}^{2}\left(\sigma^{2}+3 \mathrm{p}^{2}\right)
$$

We now suppose that the length of the Ricci operator of the perfect fluid non-flat $(\mathrm{CS})_{4}$-spacetime is $(1 / 3) r^{2}$, where $r$ is the scalar curvature of the spacetime. Then from (22) we have:

$$
\frac{1}{3} \mathrm{r}^{2}=\mathrm{k}^{2}\left(\sigma^{2}+3 \mathrm{p}^{2}\right)
$$

which yields by virtue of (16) that $\mathrm{k}^{2} \sigma(\sigma+3 \mathrm{p})=0$ Since $\sigma+3 p \neq 0$ and $k \neq 0$, it follows that $\sigma=0$ which is not possible as when the pure matter exists $\sigma$ is always 
greater than zero. Hence the spacetime under consideration cannot contain pure matter.

Now we determine the sign of pressure in such a spacetime without pure matter. Hence for $\sigma=0$, (16) implies that:

$$
\mathrm{p}=-\frac{\mathrm{r}}{3 \mathrm{k}}
$$

Again for $\sigma=0,(15)$ yields $r=6\left(\rho-\alpha^{2}\right)$ Therefore (23) reduces to

$$
p=-\frac{2}{k}\left(\rho-\alpha^{2}\right)
$$

This implies that $p>0$ if $\alpha^{2}>\rho$ and $p<0$ if $\alpha^{2}<\rho$. Thus we can state the following:

Theorem 1: If a perfect fluid non-flat $(\mathrm{CS})_{4}$-spacetime obeying Einstein's equation without cosmological constant and the square of the length of the Ricci operator is $(1 / 3) r^{2}$, then the spacetime can not contain pure matter. Moreover in such a spacetime without pure matter the pressure of the fluid is positive or negative according as:

$$
\alpha^{2}>\rho \text { or } \alpha^{2}<\rho
$$

(CS) $)_{4}$-spacetimes whose energy-momentum tensor is a codazzi tensor: This section deals with a $(\mathrm{CS})_{4}$-spacetime whose energy-momentum tensor $\mathrm{T}$ is a Codazzi tensor. Then we have:

$$
\left(\nabla_{\mathrm{X}} \mathrm{T}\right)(\mathrm{Y}, \mathrm{Z})=\left(\nabla_{\mathrm{Z}} \mathrm{T}\right)(\mathrm{Y}, \mathrm{X})
$$

We take Einstein's equation with cosmological constant given by (11). Then differentiating (11) covariantly we get:

$$
\left(\nabla_{\mathrm{X}} \mathrm{S}\right)(\mathrm{Y}, \mathrm{Z})-\frac{1}{2} \mathrm{dr}(\mathrm{X}) \mathrm{g}(\mathrm{Y}, \mathrm{Z})=\mathrm{k}\left(\nabla_{\mathrm{X}} \mathrm{T}\right)(\mathrm{Y}, \mathrm{Z})
$$

This implies

$$
\begin{aligned}
& \left(\nabla_{X} S\right)(Y, Z)-\left(\nabla_{Z} S\right)(X, Y)-\frac{1}{2} \operatorname{dr}(X) g(Y, Z) \\
& +\frac{1}{2} \operatorname{dr}(Z) g(Y, X)=k\left[\left(\nabla_{X} T\right)(Y, Z)-\left(\nabla_{Z} T\right)(Y, X)\right]
\end{aligned}
$$

By virtue of (24) and (26) we get:

$$
\begin{aligned}
\left(\nabla_{X} S\right)(Y, Z) & -\left(\nabla_{Z} S\right)(X, Y) \\
& -\frac{1}{2} \operatorname{dr}(X) g(Y, Z)+\frac{1}{2} \operatorname{dr}(Z) g(X, Y)=0
\end{aligned}
$$

Taking a frame field and contracting (27) over Y and Z, we obtain:

$$
\operatorname{dr}(X)=0 \text { for all } X
$$

Using (28) in (27) we have:

$$
\left(\nabla_{\mathrm{X}} \mathrm{S}\right)(\mathrm{Y}, \mathrm{Z})=\left(\nabla_{\mathrm{Z}} \mathrm{S}\right)(\mathrm{X}, \mathrm{Y})
$$

This leads to the following:

Theorem 2: If a $(\mathrm{CS})_{4}$-spacetime has a Codazzi type of energy-momentum tensor, then its scalar curvature is constant and its Ricci tensor is of Codazzi type.

Let $T(X, Y)=g(\tilde{T} X, Y)$. Then from (11), it follows that:

$$
\mathrm{QX}=\frac{1}{2} \mathrm{rX}+\mathrm{k} \tilde{\mathrm{T}} \mathrm{X}-\lambda \mathrm{X}
$$

where $\mathrm{Q}$ is the Ricci operator. Then (24) can be written as:

$$
\left(\nabla_{\mathrm{X}} \tilde{\mathrm{T}}\right)(\mathrm{Y})=\left(\nabla_{\mathrm{Y}} \tilde{\mathrm{T}}\right)(\mathrm{X})
$$

From (13) we have:

$$
\tilde{\mathrm{T}} \mathrm{X}=(\sigma+\mathrm{p}) \eta(\mathrm{X}) \xi+\mathrm{pX}
$$

Differentiating (32) covariantly we get:

$$
\begin{aligned}
\left(\nabla_{\mathrm{X}} \tilde{\mathrm{T}}\right)(\mathrm{Y})= & (\mathrm{X} \sigma+\mathrm{Xp}) \eta(\mathrm{Y}) \xi \\
& +(\sigma+\mathrm{p})\left(\nabla_{\mathrm{X}} \eta\right)(\mathrm{Y}) \xi \\
& +(\mathrm{Xp}) \mathrm{Y}+(\sigma+\mathrm{p}) \eta(\mathrm{Y}) \nabla_{\mathrm{X}} \xi
\end{aligned}
$$

In view of (33) we obtain by virtue of (31) that:

$$
\begin{aligned}
& (\mathrm{X} \sigma+\mathrm{Xp}) \eta(\mathrm{Y}) \xi+\alpha(\sigma+\mathrm{p}) \eta(\mathrm{Y}) \mathrm{X} \\
& +(\mathrm{Xp}) \mathrm{Y}-(\mathrm{Y} \sigma+\mathrm{Yp}) \eta(\mathrm{X}) \xi \\
& -\alpha(\sigma+\mathrm{p}) \eta(\mathrm{X}) \phi \mathrm{Y}-(\mathrm{Yp}) \mathrm{X}=0
\end{aligned}
$$

where (3) have been used.

Setting $Y=\xi$ in (34) and then using (7) we get:

$$
\alpha(\sigma+\mathrm{p}) \phi \mathrm{X}=-(\mathrm{X} \sigma) \xi-(\xi \sigma+\xi \mathrm{p}) \eta(\mathrm{X}) \xi-(\xi \mathrm{p}) \mathrm{X}
$$

Contracting (30) we obtain:

$$
\mathrm{r}=4 \lambda+(\sigma-3 \mathrm{p}) \mathrm{k}
$$

Differentiating (36) covariantly along X we have: 


$$
\operatorname{dr}(\mathrm{X})=(\mathrm{X} \sigma-3(\mathrm{Xp})) \mathrm{k}
$$

Since the spacetime under consideration has Codazzi type energy-momentum tensor, we have the relation (28). By virtue of (28) and (37) we get:

$$
(\mathrm{Xp})=\frac{1}{3}(\mathrm{X} \sigma)
$$

Using (38) in (35) we obtain:

$$
\alpha(\sigma+\mathrm{p}) \phi \mathrm{X}=-3(\mathrm{Xp}) \xi-4(\xi \mathrm{p}) \eta(\mathrm{X}) \xi-(\xi \mathrm{p}) \mathrm{X}
$$

Taking the inner product on both sides of (39) by $\xi$ we get by virtue of (7) that:

$$
\mathrm{Xp}=-(\xi \mathrm{p}) \eta(\mathrm{X})
$$

From (38) and (40), it follows that:

$$
\mathrm{X} \sigma=-(\xi \sigma) \eta(\mathrm{X})
$$

Again from (40) and (41) we have:

$$
\operatorname{grad} \mathrm{p}=-(\xi \mathrm{p}) \xi, \operatorname{grad} \sigma=-(\xi \sigma) \xi
$$

The relations (40) and (41) implies that $\mathrm{p}$ and $\sigma$ are constants over a hypersurface. This leads to the following:

Theorem 3: If the energy-momentum tensor of a perfect fluid $(\mathrm{CS})_{4}$-spacetime is a Codazzi tensor, then both the energy density and pressure of the fluid are constants over a hypersurface.

Again from (2)-(6), it follows that in a $(\mathrm{CS})_{4}$-spacetime, the following relation holds:

$$
\left(\nabla_{X} \eta\right)(Y)=\frac{1}{3} \operatorname{div} \xi[g(X, Y)+\eta(X) \eta(Y)]
$$

Since the integral curves of $\xi$ in a $(\mathrm{CS})_{4}$-spacetime are geodesics ${ }^{[7]}$, the Roy-Choudhuri equation ${ }^{[5]}$ for the fluid in a $(\mathrm{CS})_{4}$-spacetime can be written as:

$$
\begin{aligned}
\left(\nabla_{X} \eta\right)(Y)= & \omega(X, Y)+\tau(X, Y) \\
& +\frac{1}{3} \operatorname{div} \xi[g(X, Y)+\eta(X) \eta(Y)]
\end{aligned}
$$

where $\xi$ is the velocity vector field of the fluid, $\omega$ is the vorticity tensor and $\tau$ is the shear tensor respectively. Comparing (43) and (44) we get:

$$
\omega(\mathrm{X}, \mathrm{Y})+\tau(\mathrm{X}, \mathrm{Y})=0
$$

Again in a $(\mathrm{CS})_{4}$-spacetime we have $\mathrm{e}^{[7]}$ curl $\xi=0$ i.e., $\xi$ is irrotational. Hence the vorticity of the fluid vanishes. Therefore $\omega(\mathrm{X}, \mathrm{Y})=0$. Consequently (45) implies that $\tau(\mathrm{X}, \mathrm{Y})=0$. Thus we can state the following:

Theorem 4: In a perfect fluid $(\mathrm{CS})_{4}$-spacetime, the fluid has vanishing vorticity and vanishing shear.

According to Petrov ${ }^{[4]}$ classification, a spacetime can be divided into six types denoted by I, II, III, D, N and O. Again, Barnes ${ }^{[1]}$ has been proved that if a perfect fluid spacetime is shear free and vorticity free and the velocity vector field is hypersurface orthogonal and the energy density is constant over a hypersurface orthogonal to the velocity vector field, then the possible local cosmological structures of the spacetime are of Petrov type I, D or O. Since in a perfect fluid $(\mathrm{CS})_{4}$-spacetime the velocity vector field $\xi$ of the fluid is always hypersurface orthogonal ${ }^{[7]}$, by virtue of Theorem 3 and Theorem 4, we can state the following:

Theorem 5; If the energy-momentum tensor of a perfect fluid $(\mathrm{CS})_{4}$-spacetime is a Codazzi tensor, then the possible local cosmological structure of the spacetime is of Petrov type I, D or O.

Again, it can be easily seen that in a (LCS $)_{n^{-}}$ manifold(n>3) the divergence of the conformal curvature tensor $\mathrm{C}$ is given by:

$$
\begin{aligned}
(\operatorname{divC})(X, Z) Z & =\frac{n-3}{n-2}\left[\begin{array}{l}
\left(\nabla_{X} S\right)(Y, Z) \\
-\left(\nabla_{Y} S\right)(X, Z)
\end{array}\right] \\
& +\frac{n}{n-2}\left[\begin{array}{l}
\operatorname{dr}(X) g(Y, Z) \\
-\operatorname{dr}(Y) g(X, Z)
\end{array}\right]
\end{aligned}
$$

Hence if a perfect fluid (CS) $)_{4}$-spacetime is divergence free conformal curvature tensor, then (46) yields:

$$
\frac{1}{2}\left[\begin{array}{l}
\left(\nabla_{\mathrm{X}} \mathrm{S}\right)(\mathrm{Y}, \mathrm{Z}) \\
-\left(\nabla_{\mathrm{Y}} \mathrm{S}\right)(\mathrm{X}, \mathrm{Z})
\end{array}\right]+2\left[\begin{array}{l}
\mathrm{dr}(\mathrm{X}) \mathrm{g}(\mathrm{Y}, \mathrm{Z}) \\
-\mathrm{dr}(\mathrm{Y}) \mathrm{g}(\mathrm{X}, \mathrm{Z})
\end{array}\right]=0
$$

Taking an orthonormal frame field and contracting (47) over Y and Z we obtain:

$$
\operatorname{dr}(X)=0, \quad \text { for all } X
$$

Using (48) in (47) we have:

$$
\left(\nabla_{X} S\right)(Y, Z)=\left(\nabla_{Y} S\right)(X, Z)
$$

This implies that the Ricci tensor is a Codazzi tensor. Using (48) and (49) in (26) we obtain (24) and hence the energy-momentum tensor is a Codazzi tensor. This leads to the following: 
Theorem 6: If a perfect fluid $(\mathrm{CS})_{4}$-spacetime is of divergence free conformal curvature tensor, then its energy-momentum tensor is of Codazzi type.

Consequently by virtue of Theorem 5 and Theorem 6 , we can state the following:

Theorem 7: If a perfect fluid (CS) $)_{4}$-spacetime is of divergence free conformal curvature tensor, then the possible local cosmological structure of such a spacetime is of Petrov type I, D or O.

Again, Sharma ${ }^{[8]}$ proved that if a spacetime with divergence free conformal curvature tensor admits a conformal Killing vector field, then the spacetime is either conformally flat or of Petrov type N.

This leads to the following:

Theorem 8: If a perfect fluid $(\mathrm{CS})_{4}$-spacetime with divergence free conformal curvature tensor admits a conformal Killing vector field, then the spacetime is either conformally flat or of Petrov type N.

Conformally flat (CS) $)_{4}$-spacetimes: This section deals with a conformally flat $(\mathrm{CS})_{4}$-spacetime. It can be easily seen that in a conformally flat $(\mathrm{CS})_{4}$-spacetime, the Ricci tensor and curvature tensor are given by:

$$
\begin{aligned}
& \mathrm{S}(\mathrm{X}, \mathrm{Y})=\left[\frac{\mathrm{r}}{3}-\left(\rho-\alpha^{2}\right)\right] \mathrm{g}(\mathrm{X}, \mathrm{Y}) \\
&+\left[\frac{\mathrm{r}}{3}-4\left(\rho-\alpha^{2}\right)\right] \eta(\mathrm{X}) \eta(\mathrm{Y}) \\
& \mathrm{R}(\mathrm{X}, \mathrm{Y}) \mathrm{Z}= {\left[\frac{\mathrm{r}}{6}-\left(\rho-\alpha^{2}\right)\right]\left\{\begin{array}{l}
\mathrm{g}(\mathrm{Y}, \mathrm{Z}) \mathrm{X} \\
-\mathrm{g}(\mathrm{X}, \mathrm{Z}) \mathrm{Y}\}
\end{array}\right\}+} \\
& \frac{1}{2}\left[\frac{\mathrm{r}}{3}-4\left(\rho-\alpha^{2}\right)\right]\left[\begin{array}{l}
\left\{\begin{array}{l}
\mathrm{g}(\mathrm{Y}, \mathrm{Z}) \eta(\mathrm{X})- \\
\mathrm{g}(\mathrm{X}, \mathrm{Z}) \eta(\mathrm{Y})
\end{array}\right\} \xi \\
+\left\{\begin{array}{l}
\eta(\mathrm{Y}) \eta(\mathrm{Z}) \mathrm{X} \\
-\eta(\mathrm{X}) \eta(\mathrm{Z}) \mathrm{Y}
\end{array}\right\}
\end{array}\right]
\end{aligned}
$$

for all X, Y, Z.

Let $\xi^{\perp}$ be denote the 3 -dimensional distribution in a $(C S)_{4}$-spacetime orthogonal to $\xi$. Then we have $\eta(X)=$ $\eta(Y)=\eta(Z)=0$ for all $X, Y, Z \in \xi^{\perp}$. Thus from (51) we have:

$$
R(X, Y) Z=\left(\frac{r}{6}-\frac{\rho-\alpha^{2}}{2}\right)\left[\begin{array}{l}
g(Y, Z) X \\
-g(X, Z) Y
\end{array}\right]
$$

This implies that:

$$
\mathrm{R}(\mathrm{X}, \xi) \xi=-\left(\frac{\mathrm{r}}{6}-\frac{\rho-\alpha^{2}}{2}\right) X \text { for all } \mathrm{X} \in \xi^{\perp}
$$

Again, according to Karcher ${ }^{[2]}$, a Lorentzian manifold is called infinitesimally spatially isotropic relative to a unit timelike vector field $U$ if its Riemann curvature tensor $\mathrm{R}$ satisfies the relation:

$$
\mathrm{R}(\mathrm{X}, \mathrm{Y}) \mathrm{Z}=\delta[\mathrm{g}(\mathrm{Y}, \mathrm{Z}) \mathrm{X}-\mathrm{g}(\mathrm{X}, \mathrm{Z}) \mathrm{Y}]
$$

for all $\mathrm{X}, \mathrm{Y}, \mathrm{Z} \in \mathrm{U}^{\perp}$

$$
\text { and } \mathrm{R}(\mathrm{X}, \mathrm{U}) \mathrm{U}=\gamma \mathrm{X} \text { for } \mathrm{X} \in \mathrm{U}^{\perp} \text {, }
$$

where $\delta, \gamma$ are real valued functions on the manifold. Hence by virtue of (52) and (53), we can state the following:

Theorem 9: A conformally flat $(\mathrm{CS})_{4}$-spacetime is infinitesimally spatially isotropic relative to the unit timelike vector field $\xi$.

\section{REFERENCES}

1. Barnes, A., 1973. Gen. Relativ. gravit., 4: 105-129.

2. Karcher, H., 1982. Infinitesimal characterization of Friedmann Universes, Arch. Math., Basel, 38: 58-64.

3. O' Neill, B., 1983. Semi-Riemannian geometry Academic press, New York.

4. Petrov, A.Z., 1949. Einstein spaces, Pergamon press, Oxford.

5. Roy Choudhury, A.K., S. Banerjee and A. Banerjee, 1992. General relativity, Astrophysics and Cosmology, Springer Verlag, New York.

6. Shaikh, A.A., 2003. On Lorentzian almost paracontact manifolds with a structure of the concircular type, Kyungpook Math. J., 43: 305-314.

7. Shaikh, A.A. and K.K. Baishya, 2005. On concircular structure spacetimes, to appear J. Math. and Stat.

8. Sharma, R., 1993. Proper conformal symmetries of spacetimes with divergence Weyl conformal tensor. J. Math. Phys., 38: 3582-3587. 